\title{
Feedback of the electromagnetic environment on current and voltage fluctuations out of equilibrium
}

\author{
M. Kindermann, ${ }^{1}$ Yu. V. Nazarov, ${ }^{2}$ and C. W. J. Beenakker ${ }^{1}$ \\ ${ }^{1}$ Instituut-Lorentz, Universiteit Leiden, P.O. Box 9506, 2300 RA Leiden, The Netherlands \\ ${ }^{2}$ Department of Nanoscience, Delft University of Technology, Lorentzweg 1, 2628 CJ Delft, The Netherlands \\ (Received 16 June 2003; revised manuscript received 23 September 2003; published 30 January 2004)
}

\begin{abstract}
We present a theoretical framework for the statistics of low-frequency current and voltage fluctuations of a quantum conductor embedded in a linear electromagnetic environment. It takes the form of a Keldysh field theory with a generic low-frequency limit that allows for a phenomenological understanding and efficient evaluation of the statistics in the saddle-point approximation. This provides an adequate theoretical justification of our earlier calculation that made use of the so-called "cascaded Langevin approach." We show how a feedback from the environment mixes correlators of different orders. This explains the unexpected temperature dependence of the third moment of tunneling noise observed in a recent experiment. At finite temperature, current and voltage correlators of order 3 and higher are no longer linearly related. We show that a Hall bar measures voltage correlators in the longitudinal voltage and current correlators in the Hall voltage. Next, we demonstrate that the quantum high-frequency corrections to the low-frequency limit correspond to the environmental Coulomb blockade. We prove that the leading order Coulomb blockade correction to the $n$th cumulant of the current fluctuations is proportional to the voltage derivative of the $(n+1)$-th cumulant. This generalizes to any $n$ earlier results obtained for $n=1,2$.
\end{abstract}

DOI: 10.1103/PhysRevB.69.035336

PACS number(s): 73.50.Td, 05.40.-a, 73.23.-b

\section{INTRODUCTION}

A mesoscopic conductor is always embedded in a macroscopic electrical circuit that influences its transport properties. This electromagnetic environment is a source of decoherence and plays a central role for single-electron effects. ${ }^{1-5}$ It has been noticed that the quantum mechanics of the circuit can be most generally and adequately expressed in terms of a Keldysh action where the voltage drop, or corresponding phase, across the conductor is the only variable. For superconducting tunnel junctions this theory has been reviewed in Ref. 6. All information about electronic properties of the mesoscopic conductor is incorporated into the Keldysh action, which makes it non-Gaussian and nonlocal in time. Any conductor, not necessarily a mesoscopic one, can be described with a Keldysh action of similar structure.

Most transport studies transport address the time-averaged current. It is clear that time-dependent fluctuations of the electric current are also affected by the environment, which reduces the low-frequency fluctuations by a feedback loop: A current fluctuation $\delta I$ induces a counteracting voltage fluctuation $\delta V=-Z \delta I$ over the conductor, which in turn reduces the current by an amount $-G \delta V$. (Here $G$ and $Z$ are, respectively, the conductance of the mesoscopic system and the equivalent series impedance of the macroscopic voltagebiased circuit.) At zero temperature the macroscopic circuit does not generate any noise itself, and the feedback loop is the only way it affects the current fluctuations in the mesoscopic conductor, which persist at zero temperature because of the shot noise effect. ${ }^{7-9}$ In the second cumulant $C^{(2)}$, or shot-noise power, the feedback loop may be accounted for by a rescaling of the current fluctuations: $\delta I \rightarrow(1+Z G)^{-1} \delta I$. For example, the Poisson noise $C^{(2)}=e \bar{I}(1+Z G)^{-2}$ of a tunnel junction is simply reduced by a factor $(1+Z G)^{-2}$ due to the negative feedback of the series impedance.

We have recently discovered that this textbook result breaks down beyond the second cumulant. ${ }^{10}$ Terms appear which depend in a nonlinear way on lower cumulants, and which cannot be incorporated by any rescaling with powers of $1+Z G$. In the example of a tunnel junction the third cumulant at zero temperature takes the form $C^{(3)}=e^{2} \bar{I}(1$ $-2 Z G)(1+Z G)^{-4}$. This implies that the linear environment provides an important and nontrivial effect on the statistics of current and voltage fluctuations of any conductor in the low-frequency regime. In a sense, this effect is more fundamental and important than the Coulomb blockade: We will show that this environmental effect is of a classical nature and persists at $Z \ll h / e^{2}$, whereas the Coulomb blockade is the quantum correction that dissapears in the limit of small impedance.

The concrete results given in Ref. 10 were restricted to zero temperature. In Ref. 11 we removed this restriction and showed that the feedback of the electromagnetic environment on the mesoscopic conductor drastically modifies the temperature dependence of $C^{(3)}$. Earlier theory ${ }^{12-14}$ assumed an isolated mesoscopic conductor and predicted a temperature-independent $C^{(3)}$ for a tunnel junction. We showed in Ref. 11 that the coupling to the environment introduces a temperature dependence, which can even change the sign of $C^{(3)}$ as the temperature is raised. No such effect exists for the second cumulant. The temperature dependence predicted has been measured in a recent experiment. ${ }^{15} \mathrm{We}$ demonstrated in Ref. 11 that the results can be obtained in a heuristic way: under a cascade assumption one can insert by hand nonlinear terms into a Langevin equation. ${ }^{14}$ This socalled "cascaded Langevin approach" is not justified a priori. Therefore, the purpose of the present paper is to provide an adequate theoretical framework for the statistics of low- 
frequency fluctuations-a nonlinear Keldysh action that is local in time, and to support the framework with a fully quantum mechanical derivation.

The outline of this paper is as follows. In Secs. II and III we present the general framework within which we describe a broad class of electrical circuits that consist of conductors with a non-Gaussian action embedded in a macroscopic electromagnetic environment. The basis is a path integral formulation of the Keldysh approach to charge counting statistics. ${ }^{16,17}$ It allows us to evaluate correlators and crosscorrelators of currents and voltages at arbitrary contacts of the circuit. We provide an instructive interpretation of the results in terms of "pseudoprobabilities." Within this framework, we study in Secs. IV and VI series circuits of two conductors.

Further, we concentrate on the low-frequency regime and show that the path integrals over fluctuating quantum fields in this case can be readily performed in saddle-point approximation. The conditions of validity for this approximation are discussed in Sec. V. We obtain general relations between third-order correlators in a series circuit and correlators of the individual isolated conductors. We concentrate on the experimentally relevant case of a single mesoscopic conductor in series with a linear electromagnetic environment. Most experiments measure voltage correlators. In Sec. VII we propose an experimental method to obtain current correlators, using the Hall voltage in a weak magnetic field. The fundamental difference between current and voltage correlators rests on whether the variable measured is odd or even under time reversal. In Sec. VIII we show that Coulomb blockade effects due to the environment are accounted for by quantum fluctuations in our path integral. They renormalize of the low-frequency action. ${ }^{18-20}$ We conclude in Sec. IX.

\section{DESCRIPTION OF THE CIRCUIT}

We consider a circuit consisting of electrical conductors $G_{i}$, a macroscopic electromagnetic environment [with impedance matrix $Z(\omega)$ ], plus ideal current and voltage meters $M_{i}$. The current meter (zero internal impedance) is in series with a voltage source, while the voltage meter (infinite internal impedance) is in parallel to a current source. Any finite impedance of meters and sources is incorporated in the electromagnetic environment. In Fig. 1 we show examples of such circuits.

The electromagnetic environment is assumed to produce only thermal noise. To characterize this noise we consider the circuit without the mesoscopic conductors, see Fig. 2. Each pair of contacts to the environment is now attached to a current source and a voltage meter. The impedance matrix is defined by partial derivatives of voltages with respect to currents

$$
\boldsymbol{Z}=\left(\begin{array}{ll}
Z_{G G} & Z_{G M} \\
Z_{M G} & Z_{M M}
\end{array}\right)=\left(\begin{array}{ll}
\left.\frac{\partial V_{G}}{\partial I_{G}}\right|_{I_{M}} & \left.\frac{\partial V_{G}}{\partial I_{M}}\right|_{I_{G}} \\
\left.\frac{\partial V_{M}}{\partial I_{G}}\right|_{I_{M}} & \left.\frac{\partial V_{M}}{\partial I_{M}}\right|_{I_{G}}
\end{array}\right) .
$$
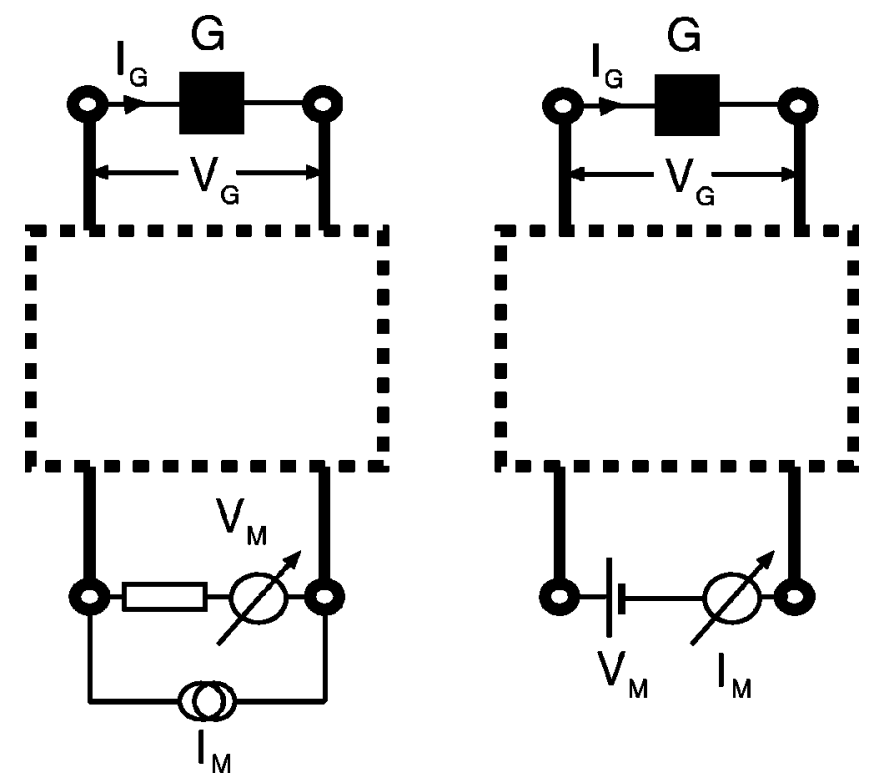

FIG. 1. Electrical circuits studied in this article. The black boxes represent conductors embedded in an electromagnetic environment (dashed rectangle). A voltage source is present at the contacts for a current measurement (right circuit) and a current source at the contacts for a voltage measurement (left circuit). The two circuits can also be combined into one larger circuit containing two conductors and both a current and a voltage meter.

(All quantities are taken at the same frequency $\omega$.) If there is more than one pair of contacts of type $G$ or $M$, then the four blocks of $\boldsymbol{Z}$ are matrices themselves. Positive and negative frequencies are related by $Z_{\alpha \beta}(-\omega)=Z_{\alpha \beta}^{*}(\omega)$. We also note

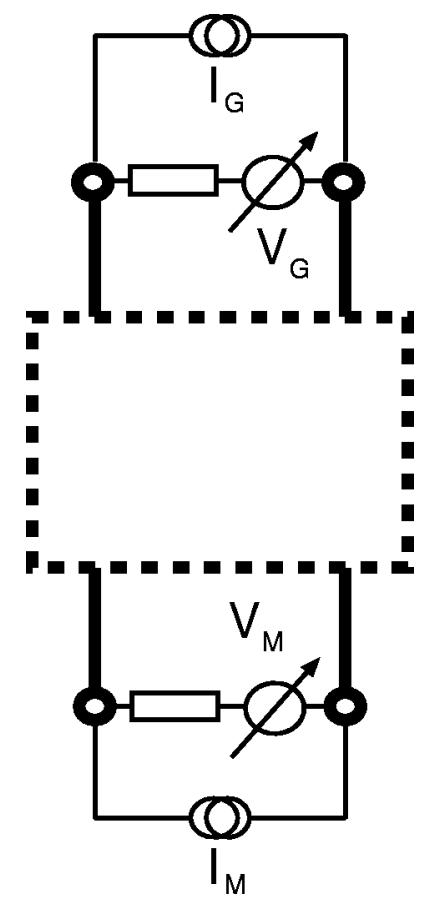

FIG. 2. Circuit used to characterize the impedance matrix of the electromagnetic environment. All contacts are now connected to a voltage meter plus a current source. 
the Onsager-Casimir ${ }^{21}$ symmetry $Z_{\alpha \beta}(B, \omega)=Z_{\beta \alpha}(-B, \omega)$, in an external magnetic field $B$. The thermal noise at each pair of contacts is Gaussian. The covariance matrix of the voltage fluctuations $\delta V_{\alpha}$ is determined by the fluctuationdissipation theorem

$$
\begin{aligned}
\left\langle\delta V_{\alpha}(\omega) \delta V_{\beta}\left(\omega^{\prime}\right)\right\rangle= & \pi \delta\left(\omega+\omega^{\prime}\right) \hbar \omega \operatorname{cotanh}\left(\frac{\hbar \omega}{2 k T}\right) \\
& \times\left[Z_{\alpha \beta}(\omega)+Z_{\beta \alpha}^{*}(\omega)\right],
\end{aligned}
$$

with $T$ the temperature of the environment.

We seek finite frequency cumulant correlators of the variables measured at the current and voltage meters

$$
\left\langle\left\langle X_{1}\left(\omega_{1}\right) \cdots X_{n}\left(\omega_{n}\right)\right\rangle\right\rangle=2 \pi \delta\left(\sum_{k=1}^{n} \omega_{k}\right) C_{\mathbf{X}}^{(n)}\left(\omega_{1}, \ldots, \omega_{n}\right) .
$$

Here $X_{i}$ stands for either $V_{M}$ or $I_{M}$. Fourier transforms are defined by $X_{i}(\omega)=\int d t \exp (i \omega t) X_{i}(t)$. Our aim is to relate the correlators at the measurement contacts to the correlators one would measure at the conductors if they were isolated from the environment.

\section{PATH INTEGRAL FORMULATION}

Correlators of currents $I_{M}$ and voltages $V_{M}$ at the measurement contacts are obtained from the generating functional

$$
\mathcal{Z}_{X}[\vec{j}]=\left\langle T_{-} e^{i \int d t\left[H+j^{-}(t) X\right]} T_{+} e^{-i \int d t\left[H+j^{+}(t) X\right]}\right\rangle .
$$

They contain moments of outcomes of measurements of the variable $X$ (equal to $I_{M}$ or $V_{M}$ ) at different instants of time. The symbols $T_{+}\left(T_{-}\right)$denote (inverse) time ordering, different on the forward and backward parts of the Keldysh contour. The exponents contain source terms $j^{ \pm}$and a Hamiltonian $H$, which we discuss separately.

The source term $j^{ \pm}(t)$ is a charge $Q_{M}=\int{ }^{t} d t^{\prime} I_{M}\left(t^{\prime}\right)$ if $X=V_{M}$, whereas it is a phase $\Phi_{M}=\int^{t} d t^{\prime} V_{M}\left(t^{\prime}\right)$ if $X$ $=I_{M}$. (We have set $\hbar$ to unity.) The superscript \pm determines on which part of the Keldysh contour the source is effective. The vector $\vec{j}=\left(j^{\mathrm{cl}}, \boldsymbol{j}^{q}\right)$ indicates the linear combinations

$$
j^{\mathrm{cl}}=\frac{1}{2} \frac{\partial}{\partial t}\left(j^{+}+j^{-}\right), \quad j^{q}=j^{+}-j^{-} .
$$

We denote vectors in this two-dimensional "Keldysh space" by a vector arrow. The "classical" source fields $\boldsymbol{j}^{\text {cl }}$ $=\left(j_{1}^{\mathrm{cl}}, j_{2}^{\mathrm{cl}}, \ldots\right)$ account for current or voltage sources at the measurement contacts. Cumulant correlators of the measured variables are generated by differentiation of $\ln \mathcal{Z}_{X}$ with respect to the "quantum" fields $\boldsymbol{j}^{q}=\left(j_{1}^{q}, j_{2}^{q}, \ldots\right)$ :

$$
\left\langle\left\langle\prod_{k=1}^{n} X_{k}\left(t_{k}\right)\right\rangle\right\rangle=\left.\prod_{k=1}^{n} \frac{\delta}{-i \delta j_{k}^{q}\left(t_{k}\right)} \ln \mathcal{Z}_{X}\right|_{j^{q}=0} .
$$

The Hamiltonian consists of three parts,

$$
H=H_{e}+\sum_{i} H_{G_{i}}-\boldsymbol{\Phi}_{G} \boldsymbol{I}_{\boldsymbol{G}}
$$

The term $H_{e}=\sum_{j} \Omega_{j} a_{j}^{\dagger} a_{j}$ represents the electromagnetic environment, which we model by a collection of harmonic oscillators at frequencies $\Omega_{j}$. The conductors connected to the environment have Hamiltonians $H_{G_{i}}$. The interaction term couples the phases $\boldsymbol{\Phi}_{\boldsymbol{G}}$ (defined by $i\left[H_{e}, \boldsymbol{\Phi}_{\boldsymbol{G}}\right]=\boldsymbol{V}_{\boldsymbol{G}}$ ) to the currents $\boldsymbol{I}_{G}$ through the conductors. The phases $\boldsymbol{\Phi}_{G}$, as well as the measured quantities $\mathbf{X}$, are linear combinations of the bosonic operators $a_{j}$ of the electromagnetic environment

$$
\begin{gathered}
\boldsymbol{\Phi}_{G}=\sum_{j}\left(c_{j}^{G} a_{j}+c_{j}^{G *} a_{j}^{\dagger}\right), \\
X=\sum_{j}\left(c_{j}^{X} a_{j}+c_{j}^{X *} a_{j}^{\dagger}\right),
\end{gathered}
$$

The coefficients $\boldsymbol{c}_{j}^{G}$ and $\boldsymbol{c}_{j}^{X}$ depend on the impedance matrix of the environment and also on which contacts are connected to a current source and which to a voltage source.

To calculate the generating functional we use a Keldysh path integral formalism. ${ }^{17,22}$ (See Appendix A for a brief introduction to this technique.) We first present the calculation for the case of a voltage measurement at all measurement contacts (so $X_{k}=V_{M_{k}}$ and $j_{k}=Q_{M_{k}}$ for all $k$ ). We will then show how the result for a current measurement can be obtained from this calculation. The path integral involves integrations over the environmental degrees of freedom $a_{j}$ weighted with an influence functional $\mathcal{Z}_{\boldsymbol{I}_{\boldsymbol{G}}}$ due to the conductors. Because the conductors are assumed to be uncoupled in the absence of the environment, this influence functional factorizes:

$$
\mathcal{Z}_{I_{G}}\left[\overrightarrow{\boldsymbol{\Phi}}_{G}\right]=\prod_{i} \mathcal{Z}_{I_{G_{i}}}\left[\vec{\Phi}_{G_{i}}\right]
$$

An individual conductor has influence functional

$$
\mathcal{Z}_{I_{G_{i}}}=\left\langle T_{-} e^{i \int d t\left[H_{G_{i}}+\Phi_{G_{i}}^{-}(t) I_{G_{i}}\right]} T_{+} e^{-i \int d t\left[H_{G_{i}}+\Phi_{G_{i}}^{+}(t) I_{G_{i}}\right]}\right\rangle .
$$

Comparing Eq. (3.8) with Eq. (3.1) for $\boldsymbol{X}=\boldsymbol{I}_{\boldsymbol{M}}$, we note that the influence functional of a conductor $G_{i}$ is just the generating functional of current fluctuations in $G_{i}$ when connected to an ideal voltage source without electromagnetic environment. That is why we use the same symbol $\mathcal{Z}$ for influence functional and generating functional.

The integrals over all environmental fields except $\overrightarrow{\boldsymbol{\Phi}}_{G}$ are Gaussian and can be done exactly. The resulting path integral expression for the generating functional $\mathcal{Z}_{V_{M}}$ takes the form

$$
\mathcal{Z}_{V_{M}}\left[\overrightarrow{\boldsymbol{Q}}_{M}\right]=\int \mathcal{D}\left[\overrightarrow{\boldsymbol{\Phi}}_{G}\right] \exp \left\{-i \mathcal{S}_{e}\left[\overrightarrow{\boldsymbol{Q}}_{M}, \overrightarrow{\boldsymbol{\Phi}}_{G}\right]\right\} \mathcal{Z}_{\boldsymbol{I}_{G}}\left[\overrightarrow{\boldsymbol{\Phi}}_{G}\right]
$$

up to a normalization constant. ${ }^{23}$ We use for the integration fields $\overrightarrow{\boldsymbol{\Phi}}_{G}$ the same vector notation as for the source fields 
$\overrightarrow{\boldsymbol{\Phi}}_{\boldsymbol{G}}=\left(\boldsymbol{\Phi}_{G}^{\mathrm{cl}}, \boldsymbol{\Phi}_{G}^{q}\right) \quad$ with $\quad \boldsymbol{\Phi}_{G}^{\mathrm{cl}}=\frac{1}{2}(\partial / \partial t)\left(\boldsymbol{\Phi}_{\boldsymbol{G}}^{+}+\boldsymbol{\Phi}_{\boldsymbol{G}}^{-}\right) \quad$ and $\boldsymbol{\Phi}_{G}^{q}$ $=\boldsymbol{\Phi}_{G}^{+}-\boldsymbol{\Phi}_{G}^{-}$. The Gaussian environmental action $\mathcal{S}_{e}$ is calculated in Appendix B. The result is given in terms of the impedance matrix $\boldsymbol{Z}$ of the environment

$$
\begin{aligned}
\mathcal{S}_{e}\left[\overrightarrow{\boldsymbol{Q}}_{M}, \overrightarrow{\boldsymbol{\Phi}}_{G}\right]= & \frac{1}{2} \int \frac{d \omega}{2 \pi}\left[\overrightarrow{\boldsymbol{Q}}_{M}^{*} \check{Z}_{M M} \overrightarrow{\boldsymbol{Q}}_{M}+\left(\overrightarrow{\boldsymbol{\Phi}}_{G}^{*}-\overrightarrow{\boldsymbol{Q}}_{M}^{*} \check{Z}_{M G}\right) \check{\boldsymbol{Y}}\right. \\
& \left.\times\left(\overrightarrow{\boldsymbol{\Phi}}_{G}-\check{\boldsymbol{Z}}_{G M} \overrightarrow{\boldsymbol{Q}}_{M}\right)\right]
\end{aligned}
$$

with the Bose-Einstein distribution $N(\omega)=[\exp (\omega / k T)$ $-1]^{-1}$. We have marked matrices in the Keldysh space by a check, for instance, $\check{\text { Y}}$.

When one substitutes Eq. (3.10) into Eq. (3.9) and calculates correlators with the help of Eq. (3.3), one can identify two sources of noise. The first source of noise is current fluctuations in the conductors that induce fluctuations of the measured voltage. These contributions are generated by differentiating the terms of $\mathcal{S}_{e}$ that are linear in $\overrightarrow{\boldsymbol{Q}}_{M}$. The second source of noise is the environment itself, accounted for by the contributions quadratic in $\vec{Q}_{M}$.

Generating functionals $\mathcal{Z}_{I_{M}}$ for circuits where currents rather than voltages are measured at some of the contacts can be obtained along the same lines with modified response functions. It is also possible to obtain them from $\mathcal{Z}_{V_{M}}$ through the functional Fourier transform derived in Appendix $C$,

$$
\mathcal{Z}_{I_{M}}\left[\vec{\Phi}_{M}\right]=\int \mathcal{D}\left[\vec{Q}_{M}\right] e^{-i \vec{Q}_{M} \times \vec{\Phi}_{M}} \mathcal{Z}_{V_{M}}\left[\vec{Q}_{M}\right]
$$

We have defined the cross product

$$
\vec{Q} \times \vec{\Phi} \equiv \int d t\left(Q^{\mathrm{cl}} \Phi^{q}-\Phi^{\mathrm{cl}} Q^{q}\right) .
$$

This transformation may be applied to any pair of measurement contacts to obtain current correlators from voltage correlators.

Equation (3.14) ensures that the two functionals

$$
\mathcal{P}[V, I]=\int \mathcal{D}[q] e^{i \int d t q V} \mathcal{Z}_{V}[\vec{Q}=(I, q)],
$$

$$
\mathcal{P}^{\prime}[V, I]=\int \mathcal{D}[\varphi] e^{i \int d t \varphi I} \mathcal{Z}_{I}[\vec{\Phi}=(V, \varphi)]
$$

are identical: $\mathcal{P}[V, I]=\mathcal{P}^{\prime}[V, I]$. This functional $\mathcal{P}$ has an intuitive probabilistic interpretation. With the help of Eq. (3.3) we obtain from $\mathcal{P}$ the correlators

$$
\begin{aligned}
\left\langle V\left(t_{1}\right) \cdots V\left(t_{n}\right)\right\rangle_{I} & =\frac{\int \mathcal{D}[V] V\left(t_{1}\right) \cdots V\left(t_{n}\right) \mathcal{P}[V, I]}{\int \mathcal{D}[V] \mathcal{P}[V, I]}, \\
\left\langle I\left(t_{1}\right) \cdots I\left(t_{n}\right)\right\rangle_{V} & =\frac{\int \mathcal{D}[I] I\left(t_{1}\right) \cdots I\left(t_{n}\right) \mathcal{P}[V, I]}{\int \mathcal{D}[I] \mathcal{P}[V, I]} .
\end{aligned}
$$

This suggests the interpretation of $\mathcal{P}[V, I]$ as a joint probability distribution functional of current and voltage fluctuations. Yet, $\mathcal{P}$ cannot properly be called a probability since it need not be positive. In the low frequency approximation introduced in the next section it is positive for normal metal conductors. However, for superconductors, it has been found to take negative values. ${ }^{24}$ It is therefore more properly called a "pseudoprobability."

We conclude this section with some remarks on the actual measurement process. The time-averaged correlators (2.3) may be measured in two different ways. In the first way the variable $X$ is measured repeatedly and results at different times are correlated afterwards. In the second way (and this is how it is usually done $\mathrm{e}^{25}$ ) one uses a detector that measures directly time integrals of $X$ (for example, by means of a 

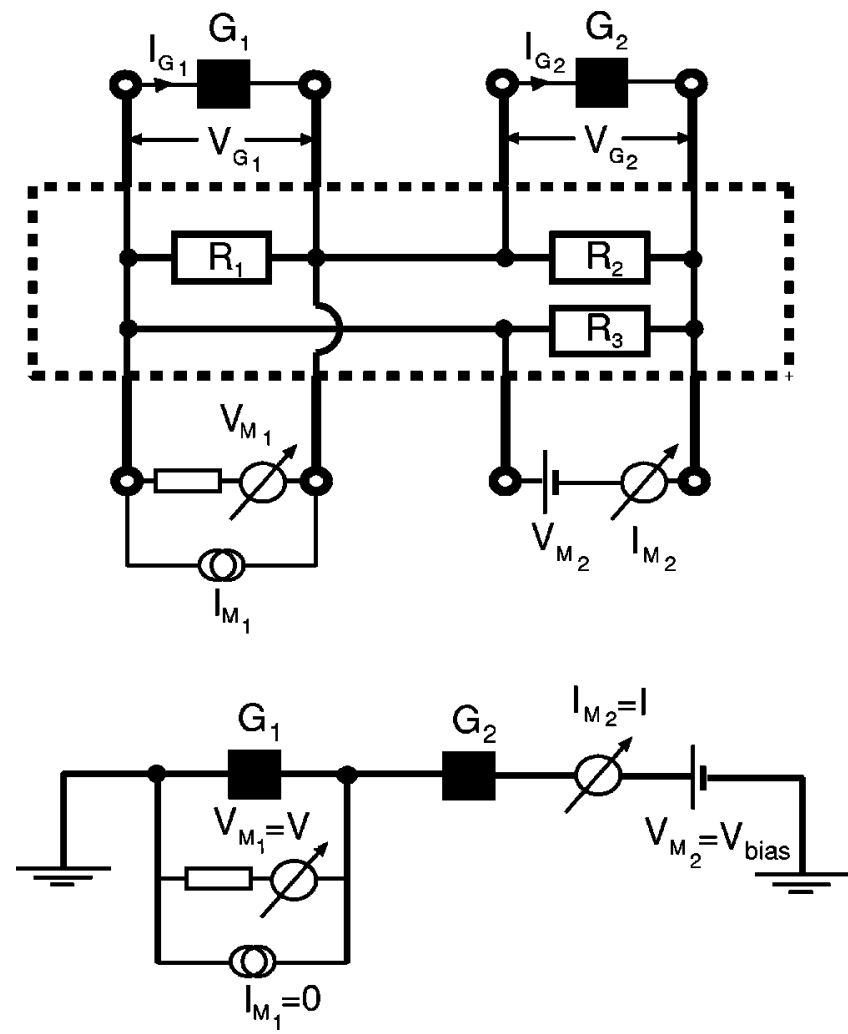

FIG. 3. Top panel: Circuit of two conductors $G_{1}, G_{2}$ in an electromagnetic environment modeled by three resistances $R_{1}, R_{2}$, $R_{3}$. In the limit $R_{1}, R_{2}, R_{3} \rightarrow \infty$ the circuit becomes equivalent to the series circuit in the lower panel.

spectral filter). The correlators measured in the first way are obtained from the generating functional according to Eq. (3.3),

$$
\begin{aligned}
2 \pi & \delta\left(\sum_{k=1}^{n} \omega_{k}\right) C_{\mathbf{X}}^{(n)}\left(\omega_{1}, \ldots, \omega_{n}\right) \\
& =\left.\prod_{k}\left[\int_{-\infty}^{\infty} d t e^{i \omega_{k} t} \frac{\delta}{-i \delta j_{k}^{q}(t)}\right] \ln \mathcal{Z}_{X}\right|_{j} q=0
\end{aligned}
$$

The second way of measurement is modeled by choosing cross-impedances that ensure that an instantaneous measurement at one pair of contacts yields a time average at another pair, for example $Z_{M G}(\omega) \propto \delta\left(\omega-\omega_{0}\right)$. The resulting frequency dependent correlators do not depend on which way of measurement one uses.

\section{TWO CONDUCTORS IN SERIES}

We specialize the general theory to the series circuit of two conductors $G_{1}$ and $G_{2}$ shown in Fig. 3 (lower panel). We derive the generating functional $\mathcal{Z}_{V, I}$ for correlators of the voltage drop $V \equiv V_{M_{1}}$ over conductor $G_{1}$ and the current $I \equiv I_{M_{2}}$ through both conductors. (The voltage drop over conductor $G_{2}$ equals $V_{M_{2}}-V_{M_{1}} \equiv V_{\text {bias }}-V$, with $V_{\text {bias }}$ the nonfluctuating bias voltage of the voltage source.) To apply the general relations of the previous section we embed the two conductors in an electromagnetic environment, as shown in the top panel of Fig. 3. In the limit of infinite resistances $R_{1}$, $R_{2}$, and $R_{3}$ this eight-terminal circuit becomes equivalent to a simple series circuit of $G_{1}$ and $G_{2}$. We take the infinite resistance limit of Eq. (3.9) in Appendix D. The result

$$
\mathcal{Z}_{V, I}[\vec{Q}, \vec{\Phi}]=\int \mathcal{D}\left[\vec{\Phi}^{\prime}\right] e^{-i \vec{\Phi}^{\prime} \times \vec{Q}} \mathcal{Z}_{1}\left[\vec{\Phi}^{\prime}\right] \mathcal{Z}_{2}\left[\vec{\Phi}-\vec{\Phi}^{\prime}\right]
$$

shows that the generating functional of current and voltage correlators in the series circuit is a functional integral convolution of the generating functionals $\mathcal{Z}_{1} \equiv \mathcal{Z}_{I_{G_{1}}}$ and $\mathcal{Z}_{2}$ $\equiv \mathcal{Z}_{I_{G_{2}}}$ of the two conductors $G_{1}$ and $G_{2}$ defined in Eq. (3.8).

Equation (4.1) implies a simple relation between the pseudo-probabilities $\mathcal{P}_{G_{1}+G_{2}}$ of the series circuit [obtained by means of Eq. (3.17) from $\left.\mathcal{Z}_{V, I}\right|_{\vec{Q}=0}$ ] and the pseudoprobabilities $\mathcal{P}_{G_{k}}$ of the individual conductors [obtained by means of Eq. (3.17) from $\mathcal{Z}_{k}$ ]. We find

$$
\mathcal{P}_{G_{1}+G_{2}}[V, I]=\int \mathcal{D} V^{\prime} \mathcal{P}_{G_{1}}\left[V-V^{\prime}, I\right] \mathcal{P}_{G_{2}}\left[V^{\prime}, I\right]
$$

This relation is obvious if one interprets it in terms of classical probabilities: The voltage drop over $G_{1}+G_{2}$ is the sum of the independent voltage drops over $G_{1}$ and $G_{2}$, so the probability $\mathcal{P}_{G_{1}+G_{2}}$ is the convolution of $\mathcal{P}_{G_{1}}$ and $\mathcal{P}_{G_{2}}$. Yet the relation (4.2) is for quantum-mechanical pseudoprobabilities.

We evaluate the convolution (4.1) in the low-frequency regime, when the functionals $\mathcal{Z}_{1}$ and $\mathcal{Z}_{2}$ become local in time,

$$
\ln \mathcal{Z}_{k}[\vec{\Phi}] \equiv-i \mathcal{S}_{k}[\vec{\Phi}]=-i \int d t S_{k}(\vec{\Phi}(t))
$$

We then do the path integration in saddle-point approximation, with the result

$$
\begin{aligned}
\ln \mathcal{Z}_{V, I}[\vec{Q}, \vec{\Phi}]= & -i \operatorname{extr}_{\left[\vec{\Phi}^{\prime}\right]}\left\{\vec{\Phi}^{\prime} \times \vec{Q}+\int d t\left[S_{1}\left(\vec{\Phi}^{\prime}(t)\right)\right.\right. \\
& \left.\left.+S_{2}\left(\vec{\Phi}(t)-\vec{\Phi}^{\prime}(t)\right)\right]\right\}
\end{aligned}
$$

The notation "extr" indicates the extremal value of the expression between curly brackets with respect to variations of $\vec{\Phi}^{\prime}(t)$. The validity of the low-frequency and saddle-point approximations is addressed in the next section.

We will consider separately the case that both conductors $G_{1}$ and $G_{2}$ are mesoscopic conductors and the case that $G_{1}$ is mesoscopic while $G_{2}$ is a macroscopic conductor. The action of a macroscopic conductor with impedance $Z$ is quadratic,

$$
\mathcal{S}_{\text {macro }}[\vec{\Phi}]=\frac{1}{2} \int \frac{d \omega}{2 \pi} \vec{\Phi}^{\dagger} \check{Y} \vec{\Phi}
$$


$\mathrm{I}(\mathrm{t})$

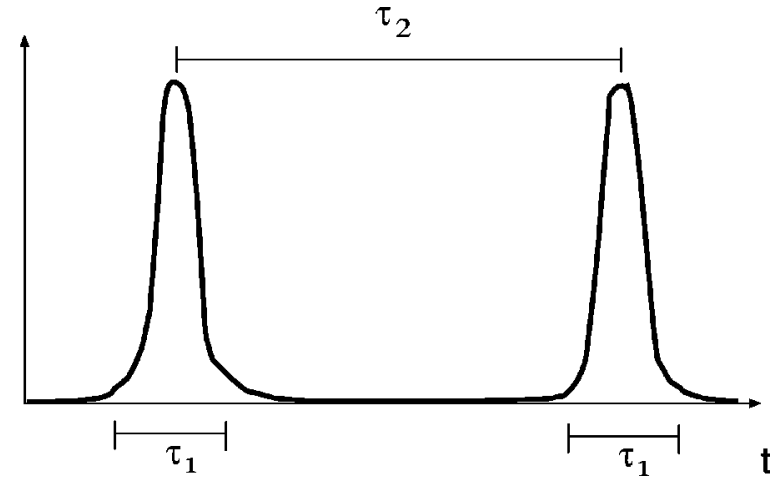

FIG. 4. Time scales of current fluctuations in a mesoscopic conductor. The time $\tau_{1}$ is the duration of current pulses, whereas $\tau_{2}$ is the mean time between subsequent current pulses.

corresponding to Gaussian current fluctuations. The matrix $\check{Y}$ is given by Eq. (3.11), with a scalar $Z_{G G}=Z$. The corresponding pseudoprobability $\mathcal{P}_{\text {macro }}$ is positive,

$$
\mathcal{P}_{\text {macro }}[V, I]=\exp \left\{-\int \frac{d \omega}{4 \pi \omega} \frac{|V-Z I|^{2}}{\operatorname{Re} Z} \tanh \left(\frac{\omega}{2 k T}\right)\right\} .
$$

Substitution of $\mathcal{P}_{\text {macro }}$ for $\mathcal{P}_{G_{2}}$ in Eq. (4.2) gives a simple result for $\mathcal{P}_{G_{1}+G_{2}}$ at zero temperature,

$$
\mathcal{P}_{G_{1}+G_{2}}[V, I]=\mathcal{P}_{G_{1}}[V-Z I, I] \text {, if } T=0 .
$$

The feedback of the macroscopic conductor on the mesoscopic conductor amounts to a negative voltage $-Z I$ produced in response to a current $I$.

The action of a mesoscopic conductor in the lowfrequency limit is given by the Levitov-Lesovik formula ${ }^{26,27}$

$$
\begin{aligned}
S_{\text {meso }}(\vec{\Phi})= & \frac{1}{2 \pi} \sum_{n=1}^{N} \int d \varepsilon \ln \left[1+\Gamma_{n}\left(e^{i e \varphi}-1\right) n_{R}\left(1-n_{L}\right)\right. \\
& \left.+\Gamma_{n}\left(e^{-i e \varphi}-1\right) n_{L}\left(1-n_{R}\right)\right],
\end{aligned}
$$

with $\vec{\Phi}=(V, \varphi)$. The $\Gamma_{n}$ 's $(n=1,2, \ldots, N)$ are the transmission eigenvalues of the conductor. The two functions $n_{L}(\varepsilon, T)=[\exp (\varepsilon / k T)+1]^{-1}$ and $n_{R}(\varepsilon, T)=n_{L}(\varepsilon+e V, T)$ are the filling factors of electron states at the left and right contacts, with $V$ the voltage drop over the conductor and $T$ its temperature.

\section{VALIDITY OF THE SADDLE-POINT APPROXIMATION}

The criterion for the applicability of the low-frequency and saddle-point approximations to the action of a mesoscopic conductor depends on two time scales (see Fig. 4). The first time scale $\tau_{1}=\min (1 / e V, 1 / k T)$ is the mean width of current pulses due to individual transferred electrons. (This time scale is known as the coherence time in optics, but in mesoscopic systems that term is used in a different context.) The second time scale $\tau_{2}=e / I \simeq\left(e^{2} / G\right) \tau_{1}$ is the mean time between current pulses.

At frequencies below $1 / \tau_{1}$ the action of the conductor becomes local in time, as expressed by Eq. (4.3). This follows from an analysis of the dependence of the action $\mathcal{S}_{k}$ on time-dependent arguments. An explicit expression for a mesoscopic conductor can be found in Ref. 20. Below the second time scale $1 / \tau_{2}$ the action of the conductor is large for values of $\vec{\Phi}$ where the nonlinearities become important. This justifies the saddle-point approximation. The nonlinearities in $\mathcal{S}_{\text {meso }}$ become relevant for $\varphi \simeq 1 / e$, so for time scales $\tau$ $\gg \tau_{2}$ we indeed have $\mathcal{S}_{\text {meso }} \simeq \tau S_{\text {meso }} \simeq \tau I \varphi \simeq \tau I / e \simeq \tau / \tau_{2} \gg 1$.

These two approximations together are therefore justified if fluctuations in the path integral (3.9) with frequencies $\omega$ above $\Lambda \simeq \min \left(1 / \tau_{1}, 1 / \tau_{2}\right)$ are suppressed. This is the case if the effective impedance of the circuit is small at high frequency: $Z(\omega) \ll h / e^{2}$ for $\omega \gtrsim \Lambda$. A small impedance acts as a heavy mass term in Eq. (4.1), suppressing fluctuations. This is seen from Eq. (4.5) for a macroscopic conductor [note that $\left.\check{Y}(\omega) \propto Z^{-1}(\omega)\right]$ and it carries over to other conductors. Physically, a small high-frequency impedance ensures that voltage fluctuations in the circuit are much slower than the electron dynamics in the conductors. Under this condition, it is sufficient to know the dynamics of the individual conductors when biased with a constant voltage, as described by Eq. (4.8). Effects of time-dependent voltage fluctuations in the circuit may then be neglected.

The same separation of time scales has been exploited in Refs. 28,29 to justify a cascaded average in the Langevin approach. We will see in Sec. VI that the results of both approaches are in fact identical in the saddle-point approximation. The two approaches differ if one goes beyond this approximation, to include the effects of a finite highfrequency impedance. Since the path integral (3.9) is microscopically justified at all time scales, it also allows us to calculate the corrections to the saddle-point solution (4.4). These corrections are usually called the "environmental Coulomb blockade." In Sec. VIII we examine the Coulomb blockade effects to lowest order in $Z(\omega)$.

\section{THIRD CUMULANTS}

\section{A. Two arbitrary conductors in series}

We use the general formula (4.4) to calculate the thirdorder cumulant correlator of current and voltage fluctuations in a series circuit of two conductors $G_{1}$ and $G_{2}$ at finite temperature. We focus on correlators at zero frequency (finite frequency generalizations are given later).

The zero-frequency correlators $C_{\mathbf{X}}^{(n)}(\bar{V})$ depend on the average voltage $\bar{V}$ over $G_{1}$, which is related to the voltage $V_{\text {bias }}$ of the voltage source by $\bar{V}=V_{\text {bias }}\left(1+G_{1} / G_{2}\right)^{-1}$. The average voltage over $G_{2}$ is $V_{\text {bias }}-\bar{V}=V_{\text {bias }}\left(1+G_{2} / G_{1}\right)^{-1}$. Our goal is to express $C_{\mathbf{X}}^{(n)}(\bar{V})$ in terms of the current correlators $\mathcal{C}_{1}^{(n)}(V)$ and $\mathcal{C}_{2}^{(n)}(V)$ that the conductors $G_{1}$ and $G_{2}$ would have if they were isolated and biased with a nonfluctuating voltage $V$. These are defined by

$$
\left\langle\left\langle I_{i}\left(\omega_{1}\right) \cdots I_{i}\left(\omega_{n}\right)\right\rangle\right\rangle_{V}=2 \pi \delta\left(\sum_{k=1}^{n} \omega_{k}\right) \mathcal{C}_{i}^{(n)}(V)
$$


where $I_{i}$ is the current through conductor $i$ at fixed voltage $V$.

To evaluate Eq. (4.4) it is convenient to discretize frequencies $\omega_{n}=2 \pi n / \tau$. The Fourier coefficients are $f_{n}$ $=\tau^{-1} \int_{0}^{\tau} d t e^{i \omega_{n} t} f(t)$. The detection time $\tau$ is sent to infinity at the end of the calculation. For zero-frequency correlators the sources at nonzero frequencies vanish and there is a saddle-point configuration such that all fields at nonzero frequencies vanish as well. We may then write Eq. (4.4) in terms of only the zero-frequency fields $\vec{\Phi}_{0}=\left(V_{0}, \varphi_{0}\right), \vec{\Phi}_{0}^{\prime}$ $=\left(V_{0}^{\prime}, \varphi_{0}^{\prime}\right)$, and $\vec{Q}_{0}=\left(I_{0}, q_{0}\right)$, with actions

$$
\tau^{-1} \mathcal{S}_{k}\left(\vec{\Phi}_{0}^{\prime}\right)=G_{k} \varphi_{0}^{\prime} V_{0}^{\prime}+i \sum_{n=2}^{\infty} \frac{\left(-i \varphi_{0}^{\prime}\right)^{n}}{n !} \mathcal{C}_{k}^{(n)}\left(V_{0}^{\prime}\right)
$$

(We assume that the conductors have a linear current-voltage characteristic.) For $\vec{\Phi}_{0}=\left(V_{\text {bias }}, 0\right)$ and $\vec{Q}_{0}=(0,0)$ the saddle point is at $\vec{\Phi}_{0}^{\prime}=(\bar{V}, 0)$. For the third-order correlators we need the extremum in Eq. (4.4) to third order in $\varphi_{0}$ and $q_{0}$. We have to expand $\mathcal{S}_{k}$ to third order in the deviation $\delta \vec{\Phi}_{0}^{\prime}$ $=\vec{\Phi}_{0}^{\prime}-(\bar{V}, 0)$ from the saddle point at vanishing sources. We have to this order

$$
\begin{aligned}
\tau^{-1} \mathcal{S}_{1}\left(\vec{\Phi}_{0}^{\prime}\right)= & G_{1} \varphi_{0}^{\prime}\left(\bar{V}+\delta V_{0}^{\prime}\right)-\frac{i}{2} \mathcal{C}_{1}^{(2)}(\bar{V}) \varphi_{0}^{\prime 2} \\
& \quad-\frac{1}{6} \mathcal{C}_{1}^{(3)}(\bar{V}) \varphi_{0}^{\prime 3}-\frac{i d}{2 d \bar{V}} \mathcal{C}_{1}^{(2)}(\bar{V}) \delta V_{0}^{\prime} \varphi_{0}^{\prime 2} \\
& +\mathcal{O}\left(\delta \vec{\Phi}_{0}^{\prime 4}\right), \\
\tau^{-1} \mathcal{S}_{2}\left(\vec{\Phi}_{0}-\vec{\Phi}_{0}^{\prime}\right) & \\
= & G_{2} \varphi_{0}^{\prime}\left(V_{\text {bias }}-\bar{V}-\delta V_{0}^{\prime}\right)-\frac{i}{2} \mathcal{C}_{2}^{(2)}\left(V_{\text {bias }}-\bar{V}\right) \varphi_{0}^{\prime 2} \\
& -\frac{1}{6} \mathcal{C}_{2}^{(3)}\left(V_{\text {bias }}-\bar{V}\right) \varphi_{0}^{\prime 3} \\
+ & \frac{i}{2} \frac{d}{d \bar{V}} \mathcal{C}_{2}^{(2)}\left(V_{\text {bias }}-\bar{V}\right) \delta V_{0}^{\prime} \varphi_{0}^{\prime 2}+\mathcal{O}\left(\delta \vec{\Phi}_{0}^{\prime 4}\right)
\end{aligned}
$$

Minimizing the sum $\mathcal{S}_{1}\left(\vec{\Phi}_{0}^{\prime}\right)+\mathcal{S}_{2}\left(\vec{\Phi}_{0}-\vec{\Phi}_{0}^{\prime}\right)$ to third order in $q_{0}$ and $\varphi_{0}$ we then find the required relation between the correlators of the series circuit and the correlators of the isolated conductors. For the second-order correlators we find

$$
\begin{gathered}
C_{I I}^{(2)}(\bar{V})=\left(R_{1}+R_{2}\right)^{-2}\left[R_{1}^{2} \mathcal{C}_{1}^{(2)}(\bar{V})+R_{2}^{2} \mathcal{C}_{2}^{(2)}\left(V_{\text {bias }}-\bar{V}\right)\right], \\
C_{V V}^{(2)}(\bar{V})=\left(R_{1}+R_{2}\right)^{-2}\left(R_{1} R_{2}\right)^{2}\left[\mathcal{C}_{1}^{(2)}(\bar{V})+\mathcal{C}_{2}^{(2)}\left(V_{\text {bias }}-\bar{V}\right)\right],
\end{gathered}
$$

$$
\begin{aligned}
C_{I V}^{(2)}(\bar{V})= & \left(R_{1}+R_{2}\right)^{-2} R_{1} R_{2}\left[R_{2} \mathcal{C}_{2}^{(2)}\left(V_{\text {bias }}-\bar{V}\right)\right. \\
& \left.-R_{1} \mathcal{C}_{1}^{(2)}(\bar{V})\right],
\end{aligned}
$$

with $R_{k}=1 / G_{k}$. The third-order correlators contain extra terms that depend on the second-order correlators

$$
\begin{aligned}
C_{I I I}^{(3)}(\bar{V})= & \left(R_{1}+R_{2}\right)^{-3}\left[R_{1}^{3} \mathcal{C}_{1}^{(3)}(\bar{V})+R_{2}^{3} \mathcal{C}_{2}^{(3)}\left(V_{\text {bias }}-\bar{V}\right)\right] \\
& +3 C_{I V}^{(2)} \frac{d}{d \bar{V}} C_{I I}^{(2)} \\
C_{V V V}^{(3)}(\bar{V})= & \left(R_{1}+R_{2}\right)^{-3}\left(R_{1} R_{2}\right)^{3}\left[\mathcal{C}_{2}^{(3)}\left(V_{\text {bias }}-\bar{V}\right)-\mathcal{C}_{1}^{(3)}(\bar{V})\right] \\
& +3 C_{V V}^{(2)} \frac{d}{d \bar{V}} C_{V V}^{(2)}, \\
C_{V V I}^{(3)}(\bar{V})= & \left(R_{1}+R_{2}\right)^{-3}\left(R_{1} R_{2}\right)^{2}\left[R_{1} \mathcal{C}_{1}^{(3)}(\bar{V})+R_{2} \mathcal{C}_{2}^{(3)}\left(V_{\text {bias }}\right.\right. \\
& -\bar{V})]+2 C_{V V}^{(2)} \frac{d}{d \bar{V}} C_{I V}^{(2)}+C_{I V}^{(2)} \frac{d}{d \bar{V}} C_{V V}^{(2)}, \\
C_{I I V}^{(3)}(\bar{V})= & \left(R_{1}+R_{2}\right)^{-3} R_{1} R_{2}\left[R_{2}^{2} \mathcal{C}_{2}^{(3)}\left(V_{\text {bias }}-\bar{V}\right)-R_{1}^{2} \mathcal{C}_{1}^{(3)}(\bar{V})\right] \\
+ & 2 C_{I V}^{(2)} \frac{d}{d \bar{V}} C_{I V}^{(2)}+C_{V V}^{(2)} \frac{d}{d \bar{V}} C_{I I}^{(2)} .
\end{aligned}
$$

These results agree with those obtained by the cascaded Langevin approach. ${ }^{11}$

\section{B. Mesoscopic and macroscopic conductor in series}

An important application is a single mesoscopic conductor $G_{1}$ embedded in an electromagnetic environment, represented by a macroscopic conductor $G_{2}$. A macroscopic conductor has no shot noise but only thermal noise. The third cumulant $\mathcal{C}_{2}^{(3)}$ is therefore equal to zero. The second cumulant $\mathcal{C}_{2}^{(2)}$ is voltage independent, given by ${ }^{8}$

$$
\mathcal{C}_{2}^{(2)}(\omega)=\omega \operatorname{cotanh}\left(\frac{\omega}{2 k T_{2}}\right) \operatorname{Re} G_{2}(\omega),
$$

at temperature $T_{2}$. We still assume low frequencies $\omega$ $\ll \max \left(e \bar{V}, k T_{1}\right)$, so the frequency dependence of $\mathcal{S}_{1}$ can be neglected. We have retained the frequency dependence of $\mathcal{S}_{2}$, because the characteristic frequency of a macroscopic conductor is typically much smaller than of a mesoscopic conductor.

From Eq. (6.6) (and a straightforward generalization to frequency-dependent correlators) we can obtain the third cumulant correlators by setting $\mathcal{C}_{2}^{(3)}=0$ and substituting Eq. (6.7). We only give the two correlators $C_{I I I}^{(3)}$ and $C_{V V V}^{(3)}$, since these are the most significant for experiments. To abbreviate the formula we denote $G=G_{1}$ and $Z(\omega)=1 / G_{2}(\omega)$. We find 


$$
\begin{gathered}
C_{I I I}^{(3)}\left(\omega_{1}, \omega_{2}, \omega_{3}\right)=\frac{\mathcal{C}_{1}^{(3)}(\bar{V})-\left(d \mathcal{C}_{1}^{(2)} / d \bar{V}\right) \sum_{j=1}^{3} Z\left(-\omega_{j}\right)\left[\mathcal{C}_{1}^{(2)}(\bar{V})-G Z\left(\omega_{j}\right) \mathcal{C}_{2}^{(2)}\left(\omega_{j}\right)\right]\left[1+Z\left(-\omega_{j}\right) G\right]^{-1}}{\left[1+Z\left(\omega_{1}\right) G\right]\left[1+Z\left(\omega_{2}\right) G\right]\left[1+Z\left(\omega_{3}\right) G\right]}, \\
-\frac{C_{V V V}^{(3)}\left(\omega_{1}, \omega_{2}, \omega_{3}\right)}{Z\left(\omega_{1}\right) Z\left(\omega_{2}\right) Z\left(\omega_{3}\right)}=\frac{\mathcal{C}_{1}^{(3)}(\bar{V})-\left(d \mathcal{C}_{1}^{(2)} / d \bar{V}\right) \sum_{j=1}^{3} Z\left(-\omega_{j}\right)\left[\mathcal{C}_{1}^{(2)}(\bar{V})+\mathcal{C}_{2}^{(2)}\left(\omega_{j}\right)\right]\left[1+Z\left(-\omega_{j}\right) G\right]^{-1}}{\left[1+Z\left(\omega_{1}\right) G\right]\left[1+Z\left(\omega_{2}\right) G\right]\left[1+Z\left(\omega_{3}\right) G\right]} .
\end{gathered}
$$

We show plots for two types of mesoscopic conductors: a tunnel junction and a diffusive metal. In both cases it is assumed that there is no inelastic scattering, which is what makes the conductor mesoscopic. The plots correspond to global thermal equilibrium $\left(T_{1}=T_{2}=T\right)$ and to a real and frequency-independent impedance $Z(\omega) \equiv Z$. We compare $C_{I}^{(3)} \equiv C_{I I I}^{(3)}$ with $C_{V}^{(3)} \equiv-C_{V V V}^{(3)} / Z^{3}$. (The minus sign is chosen so that $C_{I}^{(3)}=C_{V}^{(3)}$ at $T=0$.)

For a tunnel junction one has

$$
\mathcal{C}_{1}^{(2)}(V)=G e V \operatorname{cotanh} \frac{e V}{2 k T}, \mathcal{C}_{1}^{(3)}(V)=G e^{2} V
$$

The third cumulant of current fluctuations in an isolated tunnel junction is temperature independent, ${ }^{12}$ but this is changed
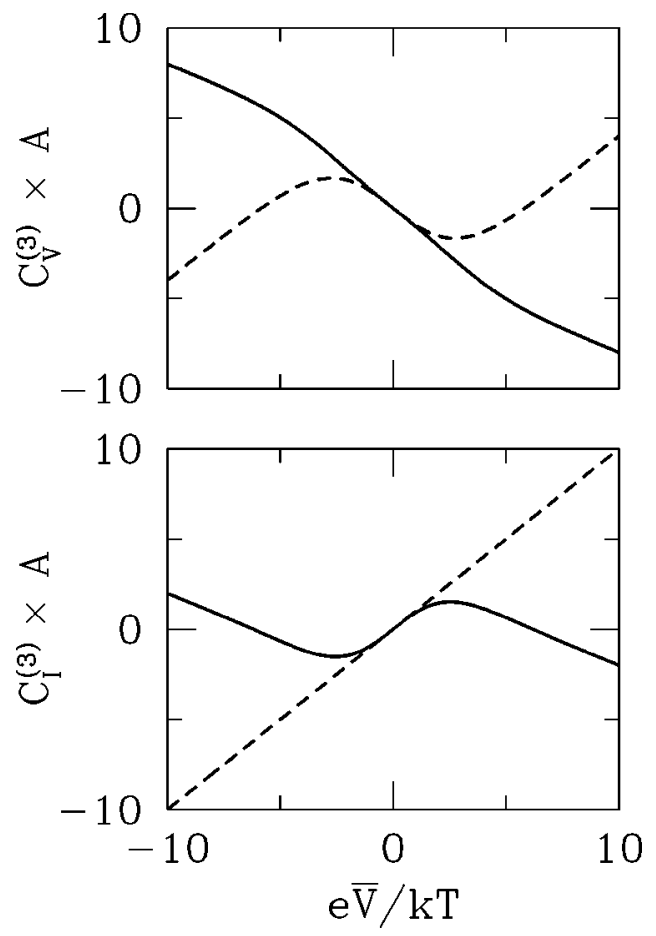

FIG. 5. Third cumulant of voltage and current fluctuations of a tunnel junction (conductance $G$ ) in an electromagnetic environment (impedance $Z$, assumed frequency independent). Both $C_{I}^{(3)}$ and $C_{V}^{(3)}$ are multiplied by the scaling factor $A=(1+Z G)^{3} / e G k T$. The two curves correspond to different values of $Z G$ (solid curve: $Z G=1$; dashed curve: $Z G=0$ ). The temperatures of the tunnel junction and its environment are chosen the same, $T_{1}=T_{2}=T$. drastically by the electromagnetic environment. ${ }^{11}$ Substitution of Eq. (6.10) into Eqs. (6.8) and (6.9) gives the curves plotted in Fig. 5 for $Z G=0$ and $Z G=1$. The slope $d C_{V}^{(3)}(\bar{V}) / d \bar{V}$ becomes strongly temperature dependent and may even change sign when $k T$ becomes larger than $e \bar{V}$. This is in qualitative agreement with the experiment of Reulet, Senzier, and Prober. ${ }^{15}$ In Ref. 15 it is shown that Eq. (6.9) provides a quantitative description of the experimental data.

For a diffusive metal we substitute the known formulas for the second and third cumulants without electromagnetic environment ${ }^{13,14}$

$$
\begin{gathered}
\mathcal{C}_{1}^{(2)}(V)=\frac{1}{3} \operatorname{GeV}(\operatorname{cotanh} p+2 / p), \\
\mathcal{C}_{1}^{(3)}(V)=e^{2} G V \frac{p\left(1-26 e^{2 p}+e^{4 p}\right)-6\left(e^{4 p}-1\right)}{15 p\left(e^{2 p}-1\right)^{2}} .
\end{gathered}
$$

We have abbreviated $p=e V / 2 k T$. Plots for $Z G=0$ and $Z G$ $=1$ are shown in Fig. 6 . The diffusive metal is a bit less striking than a tunnel junction, since the third cumulant is already temperature dependent even in the absence of the electromagnetic environment. In the limit $Z G \rightarrow \infty$ we recover the result for $C_{V}^{(3)}$ obtained by Nagaev from the cascaded Langevin approach. ${ }^{30}$

\section{HOW TO MEASURE CURRENT FLUCTUATIONS}

In Fig. 5 we have plotted both current and voltage correlators, but only the voltage correlator has been measured. ${ }^{15}$ At zero temperature of the macroscopic conductor there is no difference between the two, as follows from Eqs. (6.8) and (6.9): $C_{I I I}^{(3)}=-C_{V V V}^{(3)} / Z^{3}$ if $\mathcal{C}_{2}^{(2)}=0$, which is the case for a macroscopic conductor $G_{2}$ at $T_{2}=0$. For $T_{2} \neq 0$ a difference appears that persists in the limit of a noninvasive measurement $Z \rightarrow 0 .{ }^{11}$ Since $V$ and $I$ in the series circuit with a macroscopic $G_{2}$ are linearly related and linear systems are known to be completely determined by their response functions and their temperature, one could ask what it is that distinguishes the two measurements, or more practically: How would one measure $C_{I I I}^{(3)}$ instead of $C_{V V V}^{(3)}$ ?

To answer this question we slightly generalize the macroscopic conductor to a four-terminal, rather than two-terminal configuration, see Figs. 7 and 8 . The voltage $V_{M}$ over the extra pair of contacts is related to the current $I_{G}$ through the 

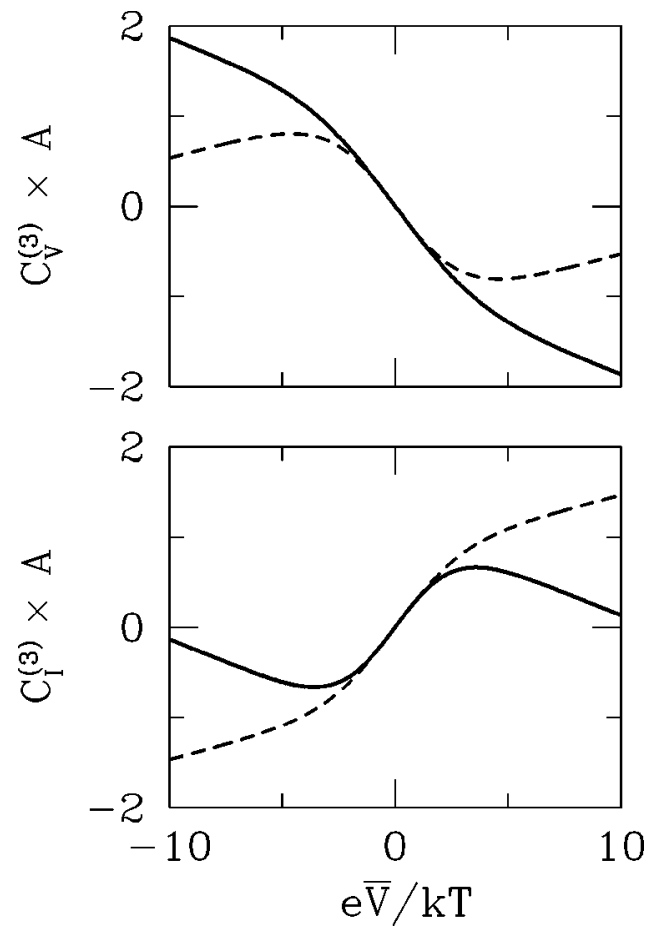

FIG. 6. Same as Fig. 5, but now for a diffusive metal.

series circuit by a cross impedance $\partial V_{M} / \partial I_{G}=Z_{M G}$. The full impedance matrix $\mathbf{Z}$ is defined as in Eq. (2.1). For simplicity we take the zero-frequency limit. For this configuration the third cumulant $C_{V_{M} V_{M} V_{M}}^{(3)}$ of $V_{M}$ is given by

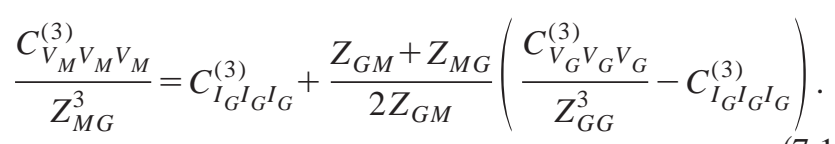

It contains the correlator $\left\langle\left\langle\delta V_{M}(\omega) \delta V_{G}\left(\omega^{\prime}\right)\right\rangle\right\rangle=2 \pi \delta(\omega$ $\left.+\omega^{\prime}\right) C_{G M}$ of the voltage fluctuations over the two pairs of terminals of the macroscopic conductor, which according to the fluctuation-dissipation theorem (2.2) is given in the zerofrequency limit by

$$
C_{G M}=k T_{2}\left(Z_{G M}+Z_{M G}\right) .
$$

The correlator $C_{G M}$ enters since $C_{V_{M} V_{M} V_{M}}^{(3)}$ depends on how thermal fluctuations in the measured variable $V_{M}$ correlate with the thermal fluctuations of $V_{G}$ which induce extra current noise in $G_{1}$.

We conclude from Eq. (7.1) that the voltage correlator $C_{V_{M} V_{M} V_{M}}^{(3)}$ becomes proportional to the current correlator

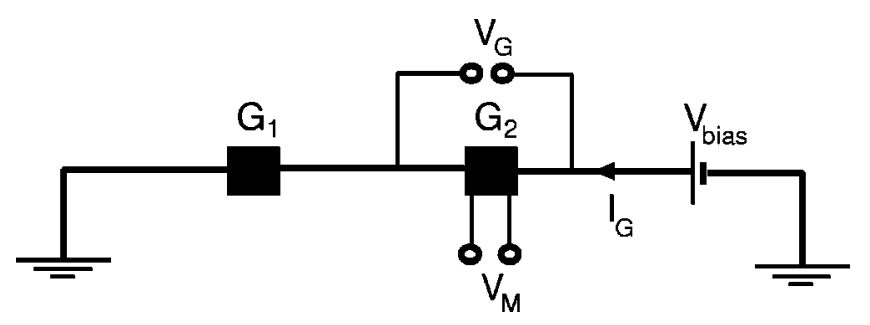

FIG. 7. Four-terminal voltage measurement.

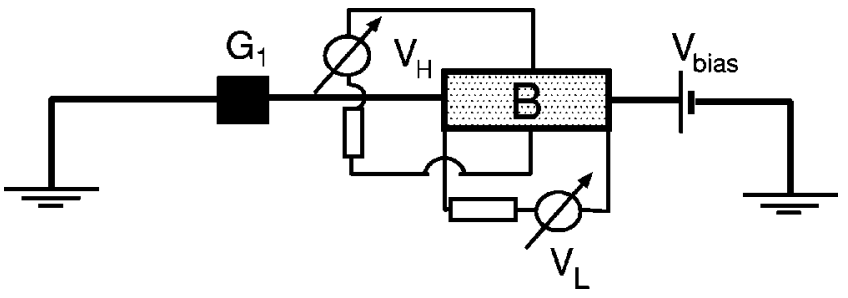

FIG. 8. Hall bar that allows one to measure the voltage correlator $C_{V}^{(3)} \propto\left\langle\left\langle V_{L}^{3}\right\rangle\right\rangle$ as well as the current correlator $C_{I}^{(3)}$ $\propto\left\langle\left\langle V_{H}^{3}\right\rangle\right\rangle$.

$C_{I_{G} I_{G} I_{G}}^{(3)}$ if $Z_{G M}+Z_{M G}=0$. This can be realized if $V_{M}$ is the Hall voltage $V_{H}$ in a weak magnetic field $B$. Then $Z_{M G}$ $=-Z_{G M}=R_{H}$, with $R_{H} \propto|B|$ the Hall resistance. The magnetic field need only be present in the macroscopic conductor $G_{2}$, so it need not disturb the transport properties of the mesoscopic conductor $G_{1}$. If, on the other hand, $V_{M}$ is the longitudinal voltage $V_{L}$, then $Z_{M G}=Z_{G M}=R_{L}$, with $R_{L}$ the longitudinal resistance. The two-terminal impedance $Z_{G G}$ is the sum of Hall and longitudinal resistances, $Z_{G G}=R_{L}$ $+R_{H}$. So one has

$$
\begin{gathered}
C_{V_{L} V_{L} V_{L}}^{(3)}=\left(\frac{R_{L}}{R_{L}+R_{H}}\right)^{3} C_{V_{G} V_{G} V_{G}}^{(3)}, \\
C_{V_{H} V_{H} V_{H}}^{(3)}=R_{H}^{3} C_{I_{G} I_{G} I_{G}}^{(3)} .
\end{gathered}
$$

One can generalize all this to an arbitrary measurement variable $X$ that is linearly related to the current $I_{G}$ through $G_{1}$. In a linear circuit the off-diagonal elements of the response tensor $\mathbf{Z}$ relating $\left(X, V_{G}\right)$ to the conjugated sources are linked by Onsager-Casimir relations. ${ }^{21}$ If $X$ is even under timereversal, then $Z_{X G}=Z_{G X}$, while if $X$ is odd, then $Z_{X G}$ $=-Z_{G X}$. In the first case $C_{X X X}^{(3)} \propto C_{V_{G} V_{G} V_{G}}^{(3)}$, while in the second case $C_{X X X}^{(3)} \propto C_{I_{G} I_{G} I_{G}}^{(3)}$.

\section{ENVIRONMENTAL COULOMB BLOCKADE}

The saddle-point approximation to the path integral (4.1) for a mesoscopic conductor $G_{1}$ in series with a macroscopic conductor $G_{2}$ (impedance $Z$ ) breaks down when the impedance at the characteristic frequency scale $\Lambda=1 / \max \left(\tau_{1}, \tau_{2}\right)$ discussed in Sec. IV is not small compared to the resistance quantum $h / e^{2}$. It can then react fast enough to affect the dynamics of the transfer of a single electron. These singleelectron effects amount to a Coulomb blockade induced by the electromagnetic environment. ${ }^{4}$ In our formalism they are accounted for by fluctuations around the saddle point of Eq. (4.1).

In Ref. 18 it has been found that the Coulomb blockade correction to the mean current calculated to leading order in $Z$ is proportional to the second cumulant of current fluctuations in the isolated mesoscopic conductor $(Z=0)$. More recently, the Coulomb blockade correction to the second cumulant of current fluctuations has been found to be proportional to the third cumulant. ${ }^{19}$ It was conjectured in Ref. 19 
that this relation also holds for higher cumulants. Here we give proof of this conjecture.

We show that at zero temperature and zero frequency the leading order Coulomb blockade correction to the $n$th cumulant of current fluctuations is proportional to the voltage derivative of the $(n+1)$-th cumulant. To extract the environmental Coulomb blockade from the other effects of the environment we assume that $Z$ vanishes at zero frequency, $Z(0)=0$. The derivation is easiest in terms of the pseudoprobabilities discussed in Sec. III.

According to Eq. (3.19), cumulant correlators of current have the generating functional

$$
\mathcal{F}_{G_{1}+G_{2}}[\vec{\Phi}=(V, \varphi)]=\ln \int \mathcal{D} I e^{-i \int d t I \varphi} \mathcal{P}_{G_{1}+G_{2}}[V, I] .
$$

Zero frequency current correlators are obtained from

$$
\left\langle\left\langle I(0)^{n}\right\rangle\right\rangle_{G_{1}+G_{2}}=\left.i^{n} \frac{\delta^{n}}{\delta[\varphi(0)]^{n}} \mathcal{F}_{G_{1}+G_{2}}[\vec{\Phi}]\right|_{\varphi=0} .
$$

We employ now Eq. (4.7) and expand $\mathcal{F}_{G_{1}+G_{2}}[\vec{\Phi}]$ to first order in $Z$,

$$
\mathcal{F}_{G_{1}+G_{2}}[\vec{\Phi}]=\mathcal{F}_{G_{1}}[\vec{\Phi}]-\frac{\int \mathcal{D} I e^{-i \int d t I \varphi} \int \frac{d \omega}{2 \pi} Z(\omega) I(\omega) \frac{\delta}{\delta V(\omega)} \mathcal{P}_{G_{1}}[V, I]}{\int \mathcal{D} I e^{-i \int d t I \varphi} \mathcal{P}_{G_{1}}[V, I]}=\mathcal{F}_{G_{1}}[\vec{\Phi}]-i \int \frac{d \omega}{2 \pi} Z(\omega) \frac{\delta^{2}}{\delta V(\omega) \delta \varphi(\omega)} \mathcal{F}_{G_{1}}[\vec{\Phi}]
$$

The last equality holds since single derivatives of $\mathcal{F}_{G_{1}}[\vec{\Phi}]$ with respect to a variable at finite frequency vanish because of time-translation symmetry. Substitution into Eq. (8.2) gives

$$
\begin{aligned}
\left\langle\left\langle I(0)^{n}\right\rangle\right\rangle_{G_{1}+G_{2}}= & \left\langle\left\langle I(0)^{n}\right\rangle\right\rangle_{G_{1}} \\
& -\int \frac{d \omega}{2 \pi} Z(\omega) \frac{\delta}{\delta V(\omega)}\left\langle\left\langle I(\omega) I(0)^{n}\right\rangle\right\rangle_{G_{1}},
\end{aligned}
$$

which is what we had set out to prove.

\section{CONCLUSION}

In conclusion, we have presented a fully quantummechanical derivation of the effect of an electromagnetic environment on current and voltage fluctuations in a mesoscopic conductor, going beyond an earlier study at zero temperature. ${ }^{10}$ The results agree with those obtained from the cascaded Langevin approach, ${ }^{11}$ thereby providing the required microscopic justification.

From an experimental point of view, the nonlinear feedback from the environment is an obstacle that stands in the way of a measurement of the transport properties of the mesoscopic system. To remove the feedback it is not sufficient to reduce the impedance of the environment. One also needs to eliminate the mixing in of environmental thermal fluctuations. This can be done by ensuring that the environment is at a lower temperature than the conductor, but this might not be a viable approach for low-temperature measurements. We have proposed here an alternative method, which is to ensure that the measured variable changes sign under time reversal. In practice this could be realized by measuring the Hall volt- age over a macroscopic conductor in series with the mesoscopic system.

The field theory developed here also provides for a systematic way to incorporate the effects of the Coulomb blockade which arise if the high-frequency impedance of the environment is not small compared to the resistance quantum. We have demonstrated this by generalizing to moments of arbitrary order a relation in the literature ${ }^{18,19}$ for the leadingorder Coulomb blockade correction to the first and second moments of the current. We refer the reader to Ref. 20 for a renormalization-group analysis of Coulomb blockade corrections of higher order.

\section{ACKNOWLEDGMENTS}

We thank D. Prober and B. Reulet for discussions of their experiment. This research was supported by the "Nederlandse organisatie voor Wetenschappelijk Onderzoek" (NWO) and by the "Stichting voor Fundamenteel Onderzoek der Materie" (FOM).

\section{APPENDIX A: KELDYSH PATH INTEGRAL}

In this appendix we give a brief introduction to the Keldysh path integral technique that we use in the text. For more details see Refs. 17,22. We restrict ourselves to a circuit with just one conductor. The Hamiltonian (3.4) reduces to

$$
H=H_{e}+H_{G}-\Phi_{G} I_{G} .
$$

We will explain how to calculate the generating functional of the phase $\Phi_{G}$. This requires the minimum amount of variables in our model, since $\Phi_{G}$ is needed anyway for the coupling of the environment circuit to the conductor. The generating function (3.1) in this case takes the form 


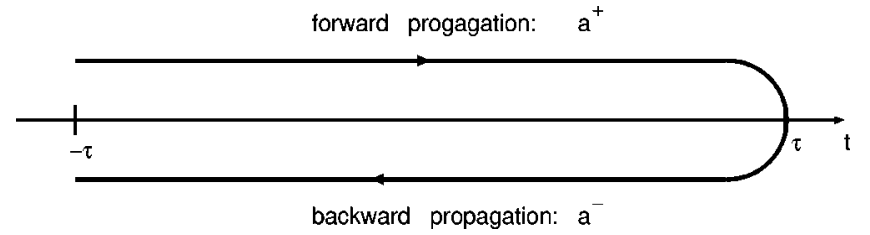

FIG. 9. Keldysh time contour with the fields $a^{+}$for forward and $a^{-}$for backward propagation.

$$
\mathcal{Z}_{\Phi_{G}}[\vec{j}]=\left\langle T_{-} e^{i \int_{-\tau}^{\tau} d t\left[H+j^{-}(t) \Phi_{G}\right]} T_{+} e^{-i \int_{-\tau}^{\tau} d t\left[H+j^{+}(t) \Phi_{G}\right]}\right\rangle
$$

(In the end we will take the limit $\tau \rightarrow \infty$.) Additionally, we restrict the analysis to an environment circuit that can be modeled by a single mode with Hamiltonian

$$
H_{e}=\Omega a^{\dagger} a, \Phi_{G}=c a+c^{*} a^{\dagger} .
$$

Here $a$ is the annihilation operator of a bosonic environmental mode and $c$ is a complex coefficient.

We first neglect the coupling of $\Phi_{G}$ to the conductor, taking $H=H_{e}$. Equation (A2) can then be rewritten as a path integral by inserting sets of coherent states (eigenstates of $a$ ), as explained, for example, in Ref. 31. In this way we introduce one time-dependent integration field $a^{+}(t)$ for the $T_{+}$-ordered time evolution operator in Eq. (A2) and a field $a^{-}(t)$ for the $T_{-}-$ordered operator. These fields propagate the system forward and backward in time, respectively. The fact that we have an integration field for forward propagation as well as one for backward propagation is characteristic for the Keldysh technique. ${ }^{32}$ Equivalently, one may formulate the theory in terms of just one field that is then defined on the so-called "Keldysh-time contour" (see Fig. 9). The contour runs from $t=-\tau$ to $t=\tau$ forward in time and backwards from $t=\tau$ to $t=-\tau$. The resulting path integral is (up to a normalization constant)

$$
\begin{aligned}
\mathcal{Z}_{\Phi_{G}[\vec{j}]=} & \int \mathcal{D} a^{ \pm} \mathcal{D} a^{ \pm *} \rho\left[a^{ \pm}(-\tau), a^{ \pm *}(-\tau)\right] \\
& \times \exp \left\{-i \int_{-\tau}^{\tau} d t\left[a^{+*}\left(-i \partial_{t}+\Omega\right) a^{+}\right.\right. \\
& +a^{-*}\left(-i \partial_{t}-\Omega\right) a^{-}+\left(c a^{+}+c^{*} a^{+*}\right) j^{+} \\
& \left.\left.-\left(c a^{-}+c^{*} a^{-*}\right) j^{-}\right]\right\}
\end{aligned}
$$

with $\rho\left[a^{+}, a^{-}\right]$the initial density matrix of the mode $a$ in the coherent state basis and $a^{+}(\tau)=a^{-}(\tau)$.

Following Feynman and Vernon, ${ }^{33}$ one can show that the coupling to the conductor in Eq. (A1) introduces an additional factor $\mathcal{Z}_{I_{G}}$ into the path integral, called the "influence functional." Instead of Eq. (A4) we then have

$$
\begin{aligned}
\left.\mathcal{Z}_{\Phi_{G}[\vec{j}]}\right] & \int \mathcal{D} a^{ \pm} \mathcal{D} a^{ \pm *} \rho\left[a^{ \pm}(-\tau), a^{ \pm *}(-\tau)\right] \\
& \times \mathcal{Z}_{I_{G}}\left[c a^{+}+c^{*} a^{+*}, c a^{-}+c^{*} a^{-*}\right] \\
& \times \exp \left\{-i \int_{-\tau}^{\tau} d t\left[a^{+*}\left(-i \partial_{t}+\Omega\right) a^{+}\right.\right. \\
& +a^{-*}\left(-i \partial_{t}-\Omega\right) a^{-}+\left(c a^{+}+c^{*} a^{+*}\right) j^{+} \\
& \left.\left.-\left(c a^{-}+c^{*} a^{-*}\right) j^{-}\right]\right\}
\end{aligned}
$$

The influence functional in our case is given by

$$
\begin{aligned}
\mathcal{Z}_{I_{G}} & {\left[\Phi_{G}^{+}, \Phi_{G}^{-}\right] } \\
& =\left\langle T_{-} e^{i \int_{-\tau}^{\tau} d t\left[H_{G}+\Phi_{G}^{-}(t) I_{G}\right]} T_{+} e^{-i \int_{-\tau}^{\tau} d t\left[H_{G}+\Phi_{G}^{+}(t) I_{G}\right]}\right\rangle .
\end{aligned}
$$

The density matrix of a thermal state of the environmental mode $a$ is the exponential of a quadratic form. Therefore the integrals over the linear combinations $c a^{ \pm}-c^{*} a^{ \pm *}$ are Gaussian and can be done exactly. With the substitution $\Phi_{G}^{ \pm}=c a^{ \pm}+c^{*} a^{ \pm *}$ and with the vector notation introduced in Sec. III we rewrite Eq. (A5) as

$$
\mathcal{Z}_{\Phi_{G}}[\vec{j}]=\int \mathcal{D} \vec{\Phi}_{G} \exp \left\{-i \mathcal{S}_{e}\left[\vec{j}, \vec{\Phi}_{G}\right]\right\} \mathcal{Z}_{I_{G}}\left[\vec{\Phi}_{G}\right]
$$

with a quadratic form $\mathcal{S}_{e}$. The more general circuits of Sec. III can be treated along the same lines, but with a multimode environmental Hamiltonian $H_{e}=\Sigma_{j} \Omega_{j} a_{j}^{\dagger} a_{j}$ and sources that couple to variables other than $\Phi_{G}$. In the limit $\tau \rightarrow \infty$ one arrives at Eq. (3.9).

\section{APPENDIX B: DERIVATION OF THE ENVIRONMENTAL ACTION}

To derive Eq. (3.10) we define a generating functional for the voltages $\mathbf{V}=\left(V_{M}, V_{G}\right)$ in the environmental circuit of Fig. 2,

$$
\mathcal{Z}_{e}[\overrightarrow{\boldsymbol{Q}}]=\left\langle T_{-} e^{i \int d t\left[H+Q^{-}(t) V\right]} T_{+} e^{-i \int d t\left[H+Q^{+}(t) V\right]}\right\rangle .
$$

We have introduced sources $Q=\left(Q_{M}, Q_{G}\right)$. Since the environmental Hamiltonian is quadratic, the generating functional is the exponential of a quadratic form in $\vec{Q}$,

$$
\mathcal{Z}_{e}[\overrightarrow{\boldsymbol{Q}}]=\exp \left(-\frac{i}{2} \int \frac{d \omega}{2 \pi} \overrightarrow{\boldsymbol{Q}}^{\dagger}(\omega) \check{\boldsymbol{G}}(\omega) \overrightarrow{\boldsymbol{Q}}(\omega)\right)
$$

The off-diagonal elements of the matrix $\check{\boldsymbol{G}}$ are determined by the impedance of the circuit 


$$
\begin{aligned}
& i \frac{\delta^{2}}{\delta Q_{\beta}^{\mathrm{cl}}\left(\omega^{\prime}\right) \delta Q_{\alpha}^{q *}(\omega)} \ln \mathcal{Z}_{e} \mid \overrightarrow{\underline{Q}}=0 \\
& =\frac{\delta}{\delta I_{\beta}\left(\omega^{\prime}\right)}\left\langle V_{\alpha}(\omega)\right\rangle=2 \pi \delta\left(\omega-\omega^{\prime}\right) Z_{\alpha \beta}(\omega) .
\end{aligned}
$$

The upper-diagonal $(c l, c l)$ elements in the Keldysh space vanish for symmetry reasons $\left(\left.\mathcal{Z}_{e}\right|_{Q^{q=0}}=0\right.$, see Ref. 22). The lower-diagonal $(q, q)$ elements are determined by the fluctuation-dissipation theorem (2.2),

$$
\begin{array}{r}
-\left.\frac{\delta^{2}}{\delta Q_{\alpha}^{q *}(\omega) \delta Q_{\beta}^{q *}\left(\omega^{\prime}\right)} \ln \mathcal{Z}_{e}\right|_{\vec{Q}=0}=\left\langle\delta V_{\alpha}(\omega) \delta V_{\beta}\left(\omega^{\prime}\right)\right\rangle \\
=\pi \delta\left(\omega+\omega^{\prime}\right) \omega \operatorname{cotanh}\left(\frac{\omega}{2 k T}\right)\left[Z_{\alpha \beta}(\omega)+Z_{\beta \alpha}^{*}(\omega)\right] .
\end{array}
$$

Consequently we have

$$
\check{\boldsymbol{G}}(\omega)=\left(\begin{array}{cc}
0 & \boldsymbol{Z}^{\dagger}(\omega) \\
\boldsymbol{Z}(\omega) & -\frac{i}{2} \omega \operatorname{cotanh}\left(\frac{\omega}{2 k T}\right)\left[\boldsymbol{Z}(\omega)+\boldsymbol{Z}^{\dagger}(\omega)\right]
\end{array}\right) .
$$

The environmental action $\mathcal{S}_{e}$ is defined by

$$
\mathcal{Z}_{e}[\overrightarrow{\boldsymbol{Q}}]=\int \mathcal{D}\left[\overrightarrow{\boldsymbol{\Phi}}_{\boldsymbol{G}}\right] \exp \left(-i \mathcal{S}_{e}\left[\overrightarrow{\boldsymbol{Q}}_{M}, \overrightarrow{\boldsymbol{\Phi}}_{\boldsymbol{G}}\right]-i \overrightarrow{\boldsymbol{\Phi}}_{\boldsymbol{G}} \times \overrightarrow{\boldsymbol{Q}}_{G}\right)
$$

One can check that substitution of Eq. (3.10) into Eq. (B6) yields the same $\mathcal{Z}_{e}$ as given by Eqs. (B2) and (B5).

\section{APPENDIX C: DERIVATION OF EQ. (3.14)}

In the limit $R \rightarrow \infty$ a voltage measurement in the circuit of Fig. 10 corresponds to a voltage measurement at contacts $M$ and $M^{\prime}$ of the circuit $C$. We obtain the generating functional $\mathcal{Z}_{V}$ of this voltage measurement from Eq. (3.9). The influence functional is now due to $C$ and it equals the generating functional $\mathcal{Z}_{I}$ of a current measurement at contacts

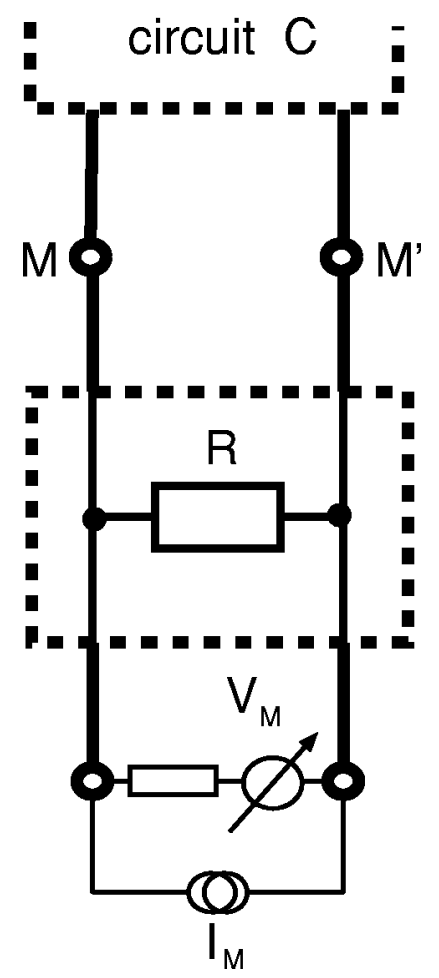

FIG. 10. Circuit to relate voltage to current measurements.

$M$ and $M^{\prime}$ of $C$. From Eq. (3.10) with $Z_{M M}=Z_{G G}=-Z_{M G}$ $=-Z_{G M}=R$ we find in the limit $R \rightarrow \infty$ that the environmental action takes the simple form $\mathcal{S}_{e}\left[\vec{Q}_{M}, \vec{\Phi}_{G}\right]=\vec{\Phi} \times \vec{Q}$, with the cross-product defined in Eq. (3.15). Consequently, we have

$$
\mathcal{Z}_{V}[\vec{Q}]=\int \mathcal{D}[\vec{\Phi}] e^{-i \vec{\Phi} \times \vec{Q}} \mathcal{Z}_{I}[\vec{\Phi}]
$$

This equation relates the generating functionals of current and voltage measurements at any pair of contacts of a circuit.

\section{APPENDIX D: DERIVATION OF EQ. (4.1)}

To derive Eq. (4.1) from Eq. (3.9) we need the environmental action $\mathcal{S}_{e}$ of the circuit shown in Fig. 3. The impedance matrix is

$$
\boldsymbol{Z}=\frac{1}{R_{1}+R_{2}+R_{3}}\left(\begin{array}{cccc}
R_{1}\left(R_{2}+R_{3}\right) & -R_{1} R_{2} & -R_{1}\left(R_{2}+R_{3}\right) & -R_{1} R_{3} \\
-R_{1} R_{2} & R_{2}\left(R_{1}+R_{3}\right) & -R_{1} R_{2} & -R_{2} R_{3} \\
-R_{1}\left(R_{2}+R_{3}\right) & -R_{1} R_{2} & R_{1}\left(R_{2}+R_{3}\right) & -R_{1} R_{3} \\
-R_{1} R_{3} & -R_{2} R_{3} & -R_{1} R_{3} & R_{3}\left(R_{1}+R_{2}\right)
\end{array}\right) .
$$

We seek the limit $R_{1}, R_{2}, R_{3} \rightarrow \infty$. The environmental action (3.10) takes the form

$$
\mathcal{S}_{e}\left[\vec{Q}_{M}, \vec{\Phi}_{G}\right]=\vec{\Phi}_{G_{1}} \times \vec{Q}_{M_{1}}+\vec{\Phi}_{G_{1}} \times \vec{Q}_{M_{2}}+\vec{\Phi}_{G_{2}} \times \vec{Q}_{M_{2}} .
$$

Substitution into Eq. (3.9) gives $\mathcal{Z}_{V V}$. Employing Eq. (3.14) to obtain $\mathcal{Z}_{V I}$ from $\mathcal{Z}_{V V}$ we arrive at Eq. (4.1). 
${ }^{1}$ E. Ben-Jacob, E. Mottola, and G. Schön, Phys. Rev. Lett. 51, 2064 (1983).

${ }^{2}$ G. Schön, Phys. Rev. B 32, 4469 (1985).

${ }^{3}$ M.H. Devoret, D. Esteve, H. Grabert, G.-L. Ingold, H. Pothier, and C. Urbina, Phys. Rev. Lett. 64, 1824 (1990).

${ }^{4}$ G.-L. Ingold and Yu. V. Nazarov, in Single Charge Tunneling, edited by H. Grabert and M.H. Devoret, Vol. 294 of NATO Advanced Studies Institute, Series B: Physics (Plenum, New York, 1992).

${ }^{5}$ H. Lee and L.S. Levitov, Phys. Rev. B 53, 7383 (1996).

${ }^{6}$ G. Schön and A.D. Zaikin, Phys. Rep. 198, 237 (1990).

${ }^{7}$ Sh. Kogan, Electronic Noise and Fluctuations in Solids (Cambridge University, Cambridge, 1996).

${ }^{8}$ Ya.M. Blanter and M. Büttiker, Phys. Rep. 336, 1 (2000).

${ }^{9}$ C.W.J. Beenakker and C. Schönenberger, Phys. Today 56, 37 (2003).

${ }^{10}$ M. Kindermann, Yu.V. Nazarov, and C.W.J. Beenakker, Phys. Rev. Lett. 90, 246805 (2003).

${ }^{11}$ C.W.J. Beenakker, M. Kindermann, and Yu.V. Nazarov, Phys. Rev. Lett. 90, 176802 (2003).

${ }^{12}$ L.S. Levitov and M. Reznikov, cond-mat/0111057 (unpublished).

${ }^{13}$ D.B. Gutman and Y. Gefen, Phys. Rev. Lett. 19, 196601 (2003).

${ }^{14}$ K.E. Nagaev, Phys. Rev. B 66, 075334 (2002).

${ }^{15}$ B. Reulet, J. Senzier, and D.E. Prober, Phys. Rev. B 69, 035302 (2004).

${ }^{16}$ Yu.V. Nazarov, Ann. Phys. (Leipzig) 8, 507 (1999).

${ }^{17}$ Yu.V. Nazarov and M. Kindermann, Eur. Phys. J. B 35, 413 (2003)

${ }^{18}$ A. Levy Yeyati, A. Martin-Rodero, D. Esteve, and C. Urbina,
Phys. Rev. Lett. 87, 046802 (2001).

${ }^{19}$ A.V. Galaktionov, D.S. Golubev, and A.D. Zaikin, Phys. Rev. B 68, 085317 (2003).

${ }^{20}$ M. Kindermann and Yu.V. Nazarov, Phys. Rev. Lett. 91, 136802 (2003).

${ }^{21}$ H.B.G. Casimir, Rev. Mod. Phys. 17, 343 (1945).

${ }^{22}$ A. Kamenev, in Strongly Correlated Fermions and Bosons in Low-Dimensional Disordered Systems, edited by I.V. Lerner, B. L. Altshuler, V.I. Fal'ko, and T. Giamarchi, Vol. 72 of NATO Science Series II (Kluwer, Dordrecht, 2002).

${ }^{23}$ M. Kindermann and Yu. V. Nazarov, in Quantum Noise in Mesoscopic Physics, edited by Yu. V. Nazarov, Vol. 97 of NATO Science Series II (Kluwer, Dordrecht, 2003).

${ }^{24}$ W. Belzig and Yu.V. Nazarov, Phys. Rev. Lett. 87, 197006 (2001).

${ }^{25}$ U. Gavish, Y. Imry, L. Levinson, and B. Yurke, in Quantum Noise in Mesoscopic Physics, edited by Yu. V. Nazarov, Vol. 97 of NATO Science Series II (Kluwer, Dordrecht, 2003).

${ }^{26}$ L.S. Levitov and G.B. Lesovik, JETP Lett. 58, 230 (1993).

${ }^{27}$ L.S. Levitov, H. Lee, and G.B. Lesovik, J. Math. Phys. 37, 4845 (1996).

${ }^{28}$ K.E. Nagaev, P. Samuelsson, and S. Pilgram, Phys. Rev. B 66, 195318 (2002).

${ }^{29}$ S. Pilgram, A.N. Jordan, E.V. Sukhorukov, and M. Büttiker, Phys. Rev. Lett. 90, 206801 (2003).

${ }^{30}$ K.E. Nagaev, cond-mat/0302008 (unpublished).

${ }^{31}$ J.W. Negele and H. Orland, Quantum Many-Particle Systems (Addison-Wesley, New York, 1988).

${ }^{32}$ J. Rammer and H. Smith, Rev. Mod. Phys. 58, 323 (1986).

${ }^{33}$ R.P. Feynman and F.L. Vernon, Ann. Phys. (N.Y.) 24, 118 (1963). 